\title{
Relaciones entre actores patrimoniales: gobernanza patrimonial, modelos neoliberales y procesos participativos ${ }^{1}$
}

Cristina Sánchez-Carretero, Guadalupe Jiménez-Esquinas | Instituto de Ciencias de Patrimonio (Incipit) del Consejo Superior de Investigaciones Científicas (CSIC)

URL de la contribución <www.iaph.es/revistaph/index.php/revistaph/article/view/3827>

Hace pocos años, un alto cargo relacionado con la gestión patrimonial de una comunidad autónoma nos interpelaba incómodo en una charla en la que analizábamos el patrimonio como un hecho metacultural, como una herramienta esencialmente política. En su argumentación defendía la preeminencia de la conservación sobre cualquier otro aspecto y la preeminencia de los especialistas que garantizan la perdurabilidad del bien sobre "opinólogos" y ciudadanos, empleando los términos de Ruiz Borrega y Marín Berral en este debate. Tres años después, la misma persona planteaba que no era posible una política patrimonial que no estuviera basada en establecer vínculos con "la ciudadanía", para lo que se necesitaban desarrollar "procesos participativos". En el debate que hemos propuesto sobre los vínculos y relaciones entre los actores implicados en el juego patrimonial buscábamos contribuciones centradas en estas transformaciones recientes en el ámbito patrimonial. Contribuciones en torno a las transformaciones silenciosas, usando la expresión del filósofo François Jullien (2010), que hacen que la participación (y sus metáforas) ocupe en la actualidad una posición central en el juego patrimonial (DAVALLON, 2010).

Como indica Alicia Castillo en este debate, "la participación está en la base de la formación del concepto actual de patrimonio cultural". Pero, a pesar de su abundante uso en el discurso, lo que se entiende y se hace bajo el paraguas de la participación varía enormemente y puede generar, y está generando, efectos muy distintos. De hecho, el lenguaje de la participación ha sido tan usado y abusado que actualmente está comenzando a ser sustituido por otros paradigmas como la cultura libre, el sharing y/o modelos de autogesión y de gobernanza de la cultura que, como analizan Perez Ponce y Pastor Pérez y Ruiz Martínez en este debate, se desa- rrollan al margen de la participación acotada y de la mano de las administraciones. Los distintos discursos, así como los tipos, grados, escalas de participación y los sistemas de clasificación que se han empleado han sido ampliamente analizados desde otras disciplinas. A través de este debate, y con el proyecto de investigación que presentamos aquí, proponemos traer el foco de análisis hacia la participación en el ámbito patrimonial no tanto con el objetivo de promocionar e implementar estos procesos de una manera acrítica, sino para entrar a cuestionarla, analizarla, debatirla y abrirla al campo de la reflexión.

La participación en el ámbito del patrimonio no sólo ha ido impregnando el lenguaje sino que también ha ido dejando huellas en legislaciones, cartas, recomendaciones y manuales desde hace décadas como en la Carta Internacional para la Gestión del Patrimonio Arqueológico (1990) de ICOMOS (art. 2 y 6); la Convención para la Salvaguarda del Patrimonio Cultural Inmaterial de la UNESCO (2003); en el manual Gestión del Patrimonio Mundial Cultural de UNESCO, ICCROM, ICOMOS, UICN (2014); en la Ley 42/2007 de Patrimonio Natural y Biodiversidad (art. 2.h y 5.2); la ley $7 / 2002$ de Ordenación Urbanística de Andalucía y la Convención sobre el Valor Patrimonial Cultural para la Sociedad del Consejo de Europa, firmada en Faro (2005) por poner algunos ejemplos.

Hace tiempo que las instituciones emprendieron una carrera por "incorporar" a la ciudadanía, una ciudadanía que ya estaba en plena carrera o que seguía otro rumbo, manifestando dichas instituciones un desfase y desactualización en los mecanismos participativos así como una voluntad por moldear los cauces para la participación. Existe una manifiesta tensión entre los instrumen- 


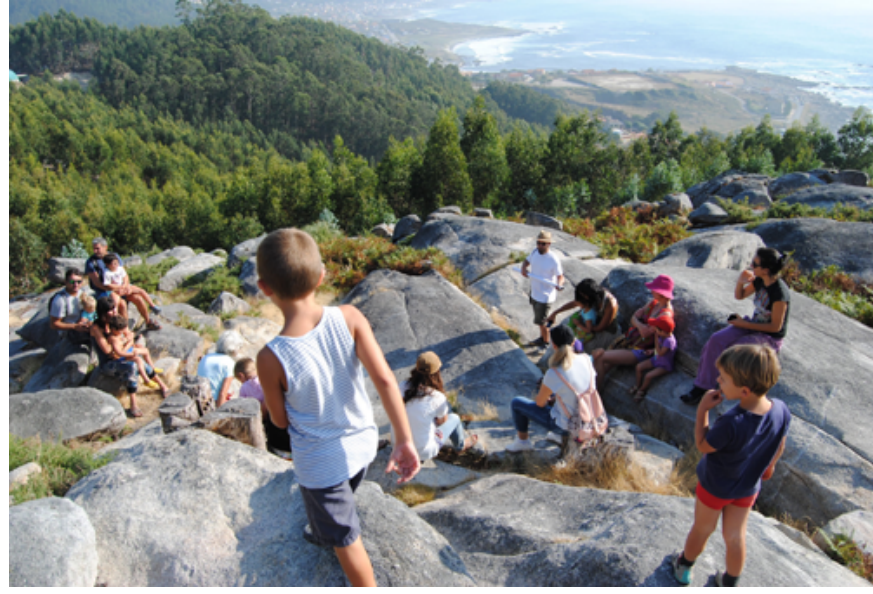

Visita guiada realizada en el proyecto Costa dos Castros, donde se están ensayando modelos alternativos de gestión arqueológica | Asociación Costa dos Castros

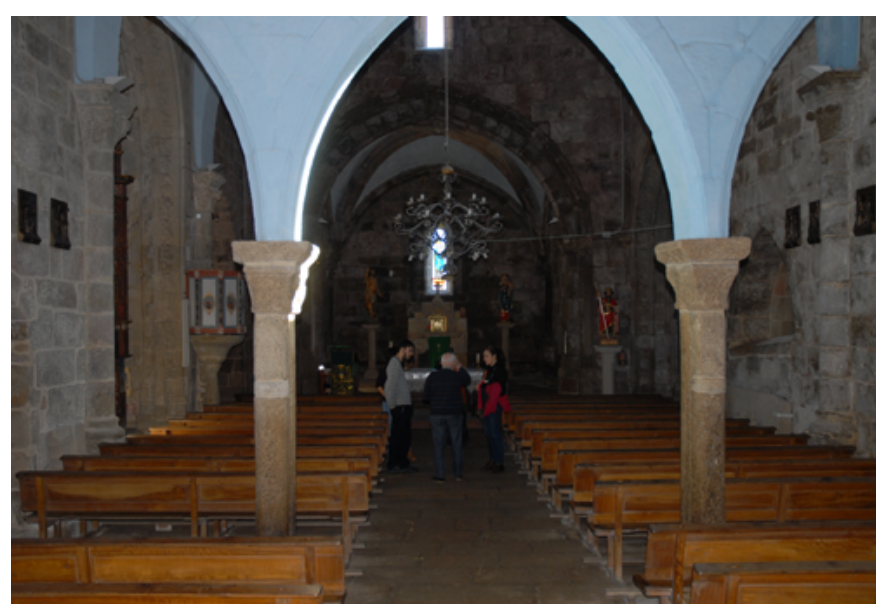

Equipo de antropología del Incipit en el interior de la iglesia de Santa María das Areas (Fisterra), donde el color azul que se empleó tras la restauración provocó protestas vecinales | Guadalupe Jiménez-Esquinas

tos participativos que se exigen a nivel legal en temas de patrimonio y la forma en la que se llevan a cabo como, por ejemplo, la necesidad de que grupos, colectivos o asociaciones se erijan en representantes y depositarios de las prácticas culturales para la presentación de las candidaturas a la UNESCO (ADELL et ál., 2015; HERTZ, 2015; URBINATI, 2011; URBINATI, 2012). De hecho, la participación ha sido incluso calificada de "nueva tiranía" por los expertos en desarrollo Cooke y Kothari (2001) al

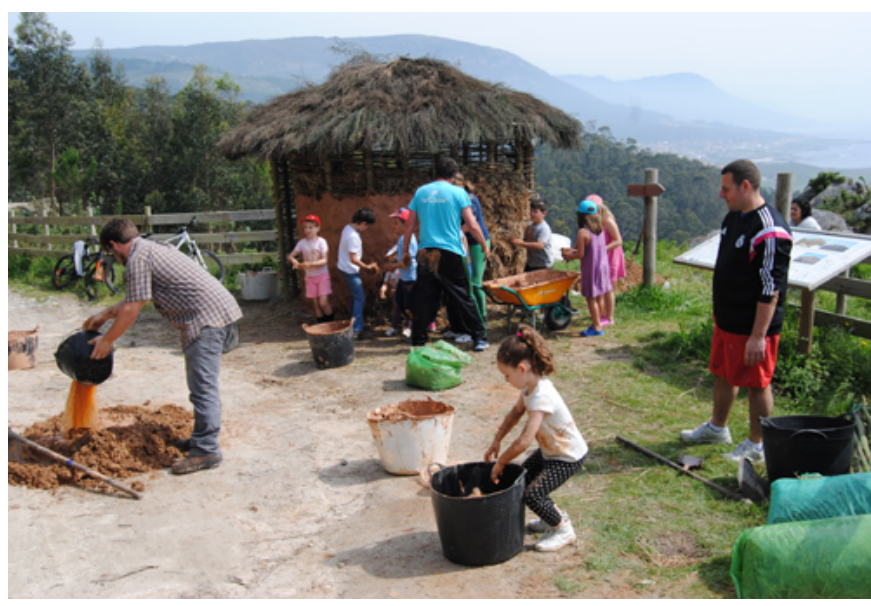

Taller de construcción de cabaña castrexa junto al yacimiento A Cabeciña, gestionado por el vecindario de la zona y el proyecto Costa dos Castros Asociación Costa dos Castros

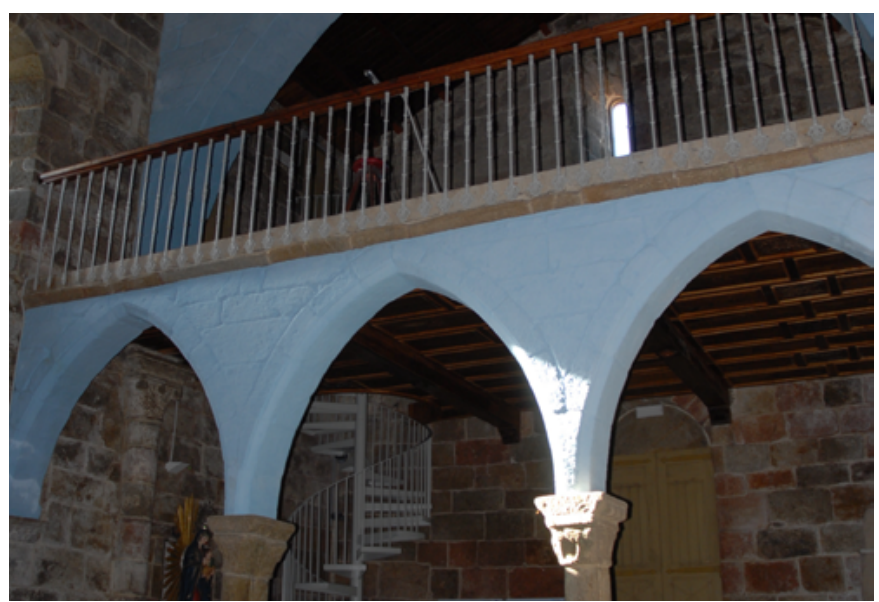

Detalle del color azul que se empleó en la última restauración de la iglesia de Santa María das Areas (Fisterra) y que provocó protestas vecinales | Guadalupe Jiménez-Esquinas

instituirse en el nuevo requisito burocrático y una herramienta para expandir la auditoría a niveles de la vida cotidiana (MACMILLAN, 2010; STRATHERN, 2000; STRATHERN, 2003). Hemos de advertir de antemano que el análisis crítico de la participación, de qué es lo que "hace" la participación y reflexionar en torno a cómo nos está construyendo o "haciendo" como sociedad, no es sinónimo de una ideología "anti-participativa" ya que, como explican los mismos autores, una cosa es anali- 
a debate Relaciones entre ciudadanía y agentes patrimoniales. Colaboración, conflicto y mediación

| coordinan Cristina Sánchez-Carretero, Guadalupe Jiménez-Esquinas

zar qué hay detrás de los discursos sobre la participación y otra es estar en su contra (COOKE; KOTHARI, 2001: 13).

En lo que se ha denominado como régimen patrimonial (HAFSTEIN, 2014; BENDIX et ál., 2012), máquina patrimonial (ALONSO GONZÁLEZ, 2013; JEUDY, 2001) o juego patrimonial (DAVALLON, 2010) la participación está siendo identificada institucionalmente como buena práctica de gobernanza. El juicio de que toda participación en patrimonio es buena y reportará beneficios se realiza de forma previa a su ejecución y, en muchas ocasiones, la evaluación de los resultados esperados no se realiza de manera sistemática. Los beneficios se suelen imaginar en forma de representación de la diversidad cultural, o por la activación de políticas identitarias, culturales y/o simbólicas pero escasamente se contempla que entren los resultados o beneficios que un proceso participativo pueda tener en la redistribución económica, la mejora de las condiciones de vida o en la toma de decisiones políticas relevantes (BENHABIB, 2006; FRASER, 1997). Tampoco se suele tener en cuenta que todo proceso de patrimonialización y de participación conlleva un trabajo, una inversión económica, de tiempo y de emociones así como también la incluye toda una serie de obligaciones como la de mantener, cuidar, preservar, transmitir y ponerlo a disposición pública, así como el propio trabajo de crear y criar las generaciones futuras que lo hereden, inputs y obligaciones que muchas veces caen en el olvido (DAVALLON, 2010; JIMÉNEZESQUINAS, en prensa).

Por esto resulta tan importante para las personas que trabajan en aspectos técnicos y de gestión en patrimonio, instituciones e investigación que la valoración del patrimonio sea inducida, creada, incentivada, forzada, que el patrimonio sea suficientemente valorado como para querer cuidarlo, modificarlo o incluso destruirlo, para que pase a formar parte del conjunto de referentes identitarios de un determinado grupo (LOWENTHAL, 2000: 22). La participación en patrimonio puede estar sirviendo como una forma de inducir que las comunidades se hagan cada vez más cargo de estas obligaciones de cuidado de manera gratuita y no reconocida de ahí que en ocasiones se recurran a las retóricas del amor o la inducción del miedo por la pérdida de los bienes o prácticas culturales, estrategias similares a las empleadas por el capital para el mantenimiento del trabajo doméstico no remunerado (JIMÉNEZ-ESQUINAS, en prensa).

La promesa de un empleo en el precarizado sector turístico es otra de las estrategias que se ponen en juego, especialmente en épocas de crisis. Esta necesidad de incitar a las personas para que cuiden el patrimonio y se impliquen en el desarrollo turístico para ahorrar tiempo, dinero y evitar conflictos se aprecia, por ejemplo, en el documento Gestión del turismo en sitios del Patrimonio Mundial: Manual práctico para administradores de sitios del Patrimonio Mundial de Arthur Pedersen y la UNESCO (2005).

La implementación de modelos participativos no es, ni mucho menos, exclusiva del campo patrimonial ya que ha estado presente desde hace más de cuarenta años en ámbitos como las políticas públicas, medio ambiente, cooperación y desarrollo (HERTZ, 2015: 26). Por ejemplo, en los años setenta el paradigma participativo llegó a ser el modelo predominante en teorías de desarrollo (CORNWALL, 2008: 269). Por otra parte, en cuanto al establecimiento de tipologías, escalas, grados y profundidades de participación, Andrea Cornwall, en su estudio sobre la participación en el ámbito del desarrollo, llega a la conclusión de que normalmente los estudios que establecen los tipos de participación son implícitamente normativos ya que sugieren una progresión desde formas "malas" de participación hacia formas más genuinas o "buenas" de participación (CORNWALL, 2008: 271). Sin embargo, proponemos trascender esta visión dicotómica y normativa para centrarnos en cómo el paradigma participativo ha sido repolitizado al servicio de una agenda neoliberal como una "tecnología de autoayuda", en términos de Adell, Bendix, Bortolotto y Tauschek (2015: 11). Como hemos dicho, no tratamos de crear pautas hacia una "buena" participación, sino de analizar cómo el régimen patrimonial está haciendo uso de los discursos de la participación, de profundizar en lo que la participación 

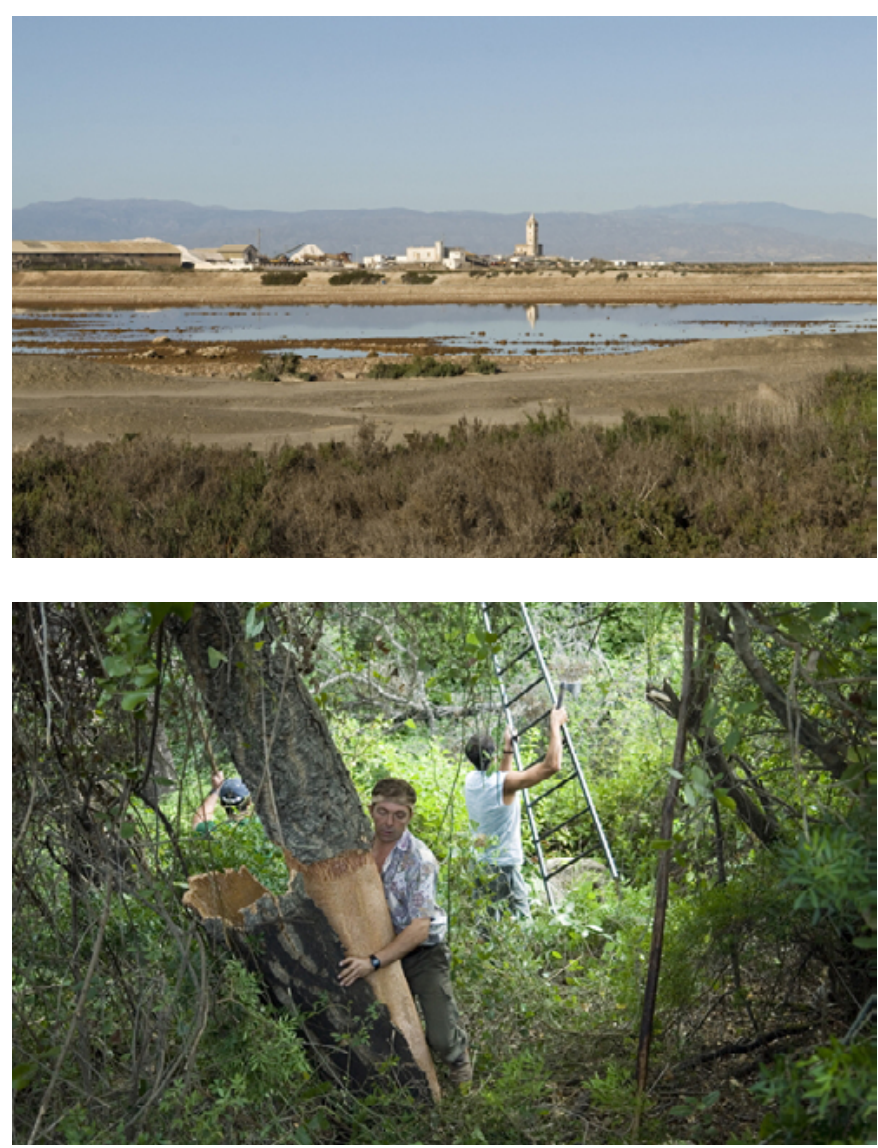

Parque Natural de Cabo de Gata-Nijar (arriba) y Aracena y Picos de Aroche (debajo), en los que se están empleando técnicas participativas en la aplicación del II Plan de Desarrollo Sostenible de Espacios Protegidos en Andalucía | fotos Fondo Gráfico IAPH (Juan Carlos Cazalla Montijano)

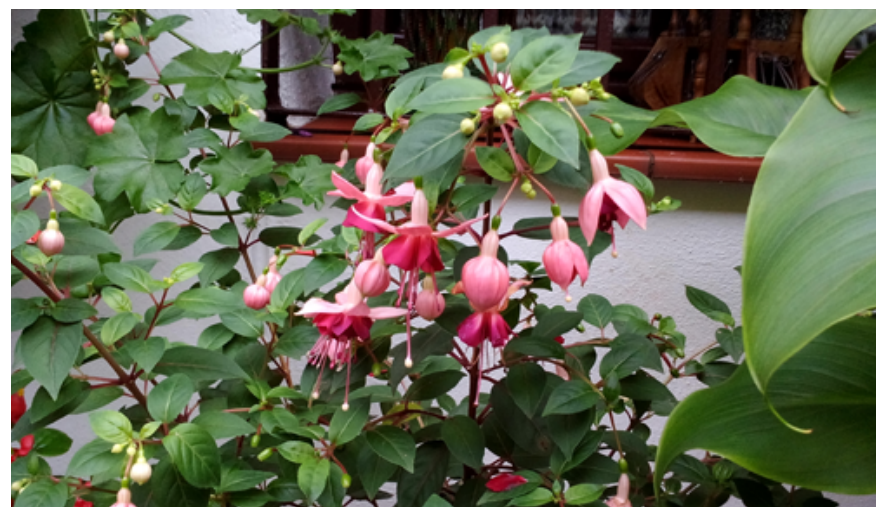

Detalle de unas flores en los patios de Córdoba, fiesta inscrita en la Lista Representativa del Patrimonio Cultural Inmaterial de la Humanidad de la UNESCO | foto Guadalupe Jiménez-Esquinas en patrimonio "hace" y "nos hace" (CORTÉS VAZQUEZ et ál., en prensa). Entre otras cosas que nos pueden hacer las instrumentalizaciones de la participación es la creación de una ciudadanía participativa que produce y reproduce el régimen patrimonial neoliberal.

Las instrumentalizaciones de los procesos participativos son un ingrediente esencial en las fracturas sociales que se producen en el seno del régimen patrimonial. Pero, además, en este debate estábamos interesadas en conocer dispositivos colaborativos e intervenciones experimentales que penetren en estas fracturas, que hiciesen del conflicto un lugar para construir diálogos y mejorar las relaciones entre actores patrimoniales a partir de su aproximación en paridad que no hiciesen más profundas las diferenciaciones maniqueas arriba/abajo, instituciones/ciudadanía, etc. Estrategias para favorecer la búsqueda de soluciones que no perjudiquen a los actores, especialmente a los más vulnerables y, sobre todo, que buscasen en las violencias y la visibilización del conflicto una fuerza para la transformación.

Partimos de la idea de que el conflicto no es algo a evitar, ya que es parte intrínseca de las relaciones humanas, sino que tiene el potencial de convertirse en una herramienta transformadora. Entre las aportaciones a este debate se pueden ver algunas propuestas en este sentido o, directamente, propuestas alternativas que apelan a la ruptura con las instituciones patrimoniales y gubernamentales como las propuestas en la plaza Taksim (analizada por Francisco Javier Navarro de Pablos) y en el barrio gòtic de Barcelona (analizado por Ana Pastor Pérez y Apen Ruíz Martínez).

La relación patrimonio-conflicto se encuentra habitualmente restringida al ámbito de la memoria histórica -en el caso de España, al patrimonio vinculado a la represión de la dictadura franquista-, como aquel patrimonio relacionado con pasados incómodos (PRATS, 2005: 26), "patrimonio negativo" (MESKELL, 2002: 558) o patrimonios difíciles (LOGAN; REEVES, 2009). Normalmente, las teorías sobre violencia y conflicto en temas de patrimonio se han aplicado a situaciones de conflictos arma- 
a debate Relaciones entre ciudadanía y agentes patrimoniales. Colaboración, conflicto y mediación

| coordinan Cristina Sánchez-Carretero, Guadalupe Jiménez-Esquinas

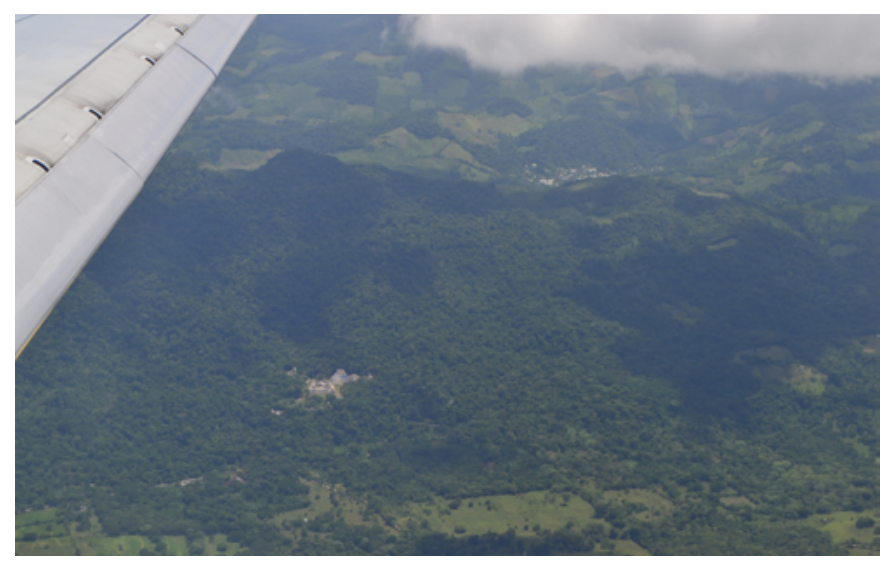

Vista aérea de la zona arqueológica y parque nacional de Palenque, Chiapas (México), en la que se desarrolla uno de los estudios de caso del proyecto ParticiPat | foto Amilcar Vargas

dos y los eufemísticamente llamados post-conflictos armados. Como indicábamos en el resumen del debate, aquí nos referimos a conflictos vinculados a la vida diaria, cotidiana, de "baja intensidad" o "bajo impacto" en cuanto que no están vinculados a situaciones de conflictos armados, pero que se dan en todo el ámbito del patrimonio como arena política y escenario de luchas ideológicas.

El conflicto surge por la intrínseca naturaleza "disonante" del patrimonio (TUNBRIDGE; ASHWORTH, 1996): el patrimonio refuerza las instancias de poder que lo crean y potencialmente deshereda o no incluye a todo el mundo. El patrimonio es disonante porque no produce resonancias (DELEUZE, 1994: 98) con otras personas, no se ubica en la frecuencia de voz en la que las personas ordinarias hablan o sienten (MUÑOZ, 2006: 677), no afecta a personas que no han creado el patrimonio por y para ellas, o alejadas de instancias de poder. Es el propio discurso patrimonial autorizado y las prácticas patrimoniales habituales las que producen unas fracturas sociales estructurales que se reproducen por la propia maquinaria del sistema, independientemente del elemento patrimonializable. Más allá de los estallidos de violencia subjetiva, como el que se produjo en el caso de Muxía, se sigue produciendo una violencia objetiva, estructural y constante (ŽIŽEK, 2008) que está

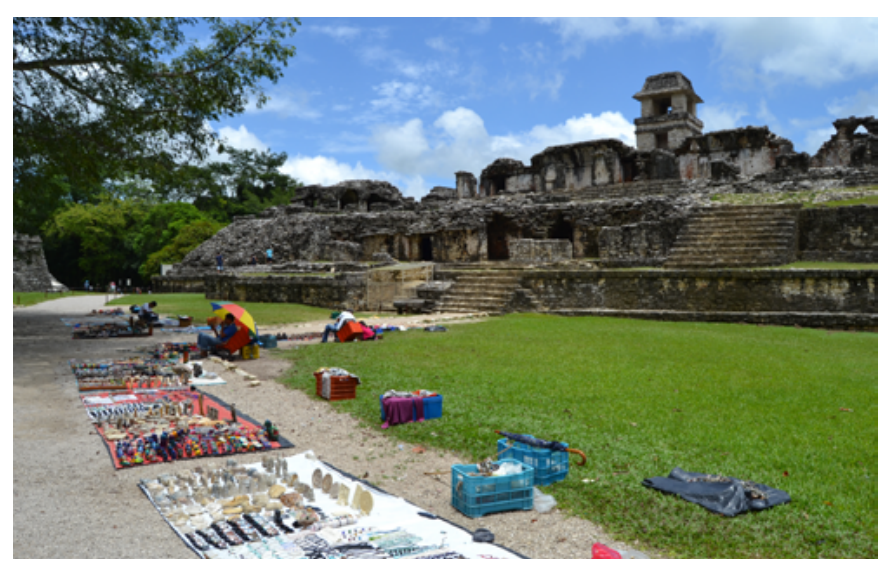

Participación de las comunidades locales en la gestión de los sitios arqueológicos Patrimonio Mundial. Vendedores dentro de la zona arqueológica de Palenque (México) | foto Amilcar Vargas

lejos de encontrar su vía de solución con la implementación de medidas de divulgación del patrimonio, ya al final de la cadena de la cadena interpretativa o de valor del patrimonio (CRIADO-BOADO, 1996; CRIADO-BOADO; BARREIRO 2013) o, según las últimas tendencias, que las comunidades se hagan cargo de su cuidado y conservación a largo plazo.

Hace poco tiempo analizábamos en un artículo publicado en esta revista el conflicto surgido a raíz de la restauración de una iglesia (JIMÉNEZ-ESQUINAS; SÁNCHEZCARRETERO, 2015). Después de evidenciar las jerarquías que se establecían entre discursos relacionados con el patrimonio, las fracturas que se creaban, las distancias y las sorderas forzadas, en ese mismo artículo proponíamos la figura de la mediación para la resolución de conflictos patrimoniales (SÁNCHEZ-CARRETERO, 2012).

Existen distintos modelos teóricos y prácticos de mediación cultural como la que se emplea en el museo Reina Sofía o en Medialab-Prado, que tratan de aproximar contenidos culturales de una forma horizontal. Sin embargo la propuesta experimental que estamos empezando a explorar se aleja de estos modelos ya que se orienta a la resolución de conflictos con una metodología que mezcla las experiencias de distintas disciplinas y enfoques, 
sobre todo la investigación acción participativa. Partimos de la idea de que el modelo de mediación social es un aparato ideológico al servicio de las instituciones para que no aparezcan como evidentes las contradicciones del discurso patrimonial autorizado. Desde esta premisa, pretendemos crear una herramienta que haga visible el juego de las relaciones de poder que se establecen entre las personas, asociaciones, instituciones, e incluso entre seres no humanos, a los que se les confiere poder-que se podrían entender como actantes, por ejemplo los planes directores o leyes de patrimonio-. Esta herramienta facilitadora o mediadora es parte del proyecto de investigación Participat que incluye varios estudios de caso.

Cerramos nuestra contribución a este debate presentando el proyecto ParticiPat que se propone analizar las instrumentalizaciones de la participación en gestión patrimonial. Este proyecto, financiado dentro del Plan Nacional, se centra en nueve estudios de caso en los que se aplica el método etnográfico para analizar diferentes realidades en las que se vinculan los tres polos que consideramos centrales para entender la transformación que se está dando: la relación entre gobernanza patrimonial, modelos neoliberales y procesos participativos.

El proyecto "Patrimonio y participación social: propuesta metodológica y revisión crítica, ParticiPat, (HAR201454869-R)" se centra en el uso de procesos participativos en procesos de patrimonialización. Se plantea un triple objetivo: (1) analizar el papel que están jugando los procesos participativos en políticas patrimoniales; (2) analizar el vínculo entre movimientos sociales y procesos de patrimonialización; (3) analizar el vínculo entre dichos movimientos e instituciones patrimoniales. El contexto de la actual situación de crisis hace que sea especialmente relevante la elaboración de nuevas propuestas sobre la función del patrimonio. A su vez, este contexto de crisis está provocando nuevas fórmulas de participación en patrimonio y fomentando la aparición de modelos alternativos de gestión patrimonial muy interesantes, pero también la participación se está instrumentalizando desde los discursos patrimoniales autorizados. Pensamos que un paso previo al abordaje de los mis- mos, es conocer los usos, ausencia de usos e instrumentalizaciones de los procesos participativos en los procesos de patrimonialización, ya que investigaciones previas sobre la implementación de procesos participativos en procesos de patrimonialización han señalado su uso cosmético (BENDIX et ál., 2012; COCA, 2008; QUINTERO MORÓN, 2011; SÁNCHEZ-CARRETERO, 2012; DURÁN SALADO, 2007).

Los estudios de caso incluyen un amplio repertorio de procesos de patrimonialización: desde parques naturales, a patrimonio inmaterial pasando por grandes conjuntos arqueológicos patrimonio mundial, pequeños proyectos de excavación en comunidad donde se ensayan modelos de gestión alternativos y microestudios de conflictos surgidos a raíz de restauraciones de iglesias. Desde el punto de partida de este proyecto proponemos comparar y someter a las mismas preguntas de investigación a casos de estudio que son aparentemente dispares pero que guardan elementos en común. De hecho las hipótesis de partida son (1) que el régimen patrimonial está creando fracturas sociales que se reproducen independientemente del tipo de patrimonio del que se trate y (2) estas fracturas están en mucho casos vinculadas a las instrumentalizaciones de procesos participativos.

\section{Los nueve estudios de caso son:}

> Usos de técnicas participativas en la aplicación del II Plan de Desarrollo Sostenible de Espacios Protegidos en Andalucía: Parque Natural de Cabo de Gata-Nijar y de Aracena y Picos de Aroche. Llevado a cabo por José Antonio Cortés (Universidad de Sheffield).

> Participación y gestión de recursos en el Parque Natural Fuentes del Narcea, Degaña e Ibias (Asturias). Llevado a cabo por Pablo Alonso González (Incipit, CSIC) y David González (Incipit, CSIC).

$>$ Asociacionismo y participación en el Camino de Santiago. Llevado a cabo por Nieves Herrero (Universidad de Santiago de Compostela).

> Usos de técnicas participativas y participación de "neoandalusíes" en el Festival Islámico de Mértola, Alentejo, Portugal. Llevado a cabo por Virtudes Téllez 


\title{
perspectivas
}

\author{
a debate Relaciones entre ciudadanía y agentes patrimoniales. Colaboración, conflicto y mediación \\ | coordinan Cristina Sánchez-Carretero, Guadalupe Jiménez-Esquinas
}

(Universidad de Castilla-La Mancha).

$>$ Proceso participativo vinculado a las protestas después de la obras de restauración en la iglesia de Santa María das Areas en Fisterra. Llevado a cabo por Cristina Sánchez-Carretero (Incipit, CSIC) y Guadalupe Jiménez-Esquinas (Incipit, CSIC).

$>$ Modelos alternativos de gestión arqueológica: el caso de Costa dos Castros (Galicia). Llevado a cabo por Guadalupe Jiménez-Esquinas (Incipit, CSIC).

$>$ Los patios de Córdoba. Llevado a cabo por Victoria Quintero Morón (Universidad Pablo de Olavide).

$>$ Participación de las comunidades locales en la gestión de los sitios arqueológicos Patrimonio Mundial en México. Llevado a cabo por Amílcar Vargas (Universidad de Barcelona).

$>$ Movimientos sociales y modelos patrimoniales alternativos. Llevado a cabo por Joan Roura (Incipit, CSIC)

Además de las personas que desarrollan los estudios de caso, existe un grupo de personas que participan en el proyecto: Antonio Díaz Aguilar (Universidad Pablo de Olavide), Ana Ruiz Blanch (Incipit, CSIC), José Muñoz Albaladejo (Incipit, CSIC), David Barreiro (Incipit, CSIC), Gema Carrera Díaz (IAPH) y María Masaguer Otero (Estudio Rurban). El proyecto se coordina desde el Incipit y la propuesta inicial -que ha variado sustancialmente- se puede consultar en http://digital.csic.es/ handle/10261/116297.

En este proyecto, que empezó en septiembre de 2015 y finalizará en diciembre de 2017, nos planteamos los temas que hemos trasladado a este debate y que hemos expuesto en la parte inicial de este ensayo: las formas en las que se usan los procesos participativos en la gestión patrimonial; los usos cosméticos que se han detectado; los conflictos, fracturas y violencia estructural que encierran; los intereses y retornos que subyacen a las movilizaciones sociales en defensa (o en contra) del patrimonio; los tipos de intereses y retornos que están legitimados socialmente: identitarios, afectivos, económicos, laborales, políticos. Todos estos temas son parte esencial de la transformación silenciosa que atraviesa el patrimonio y son elementos clave para evitar que los procesos participativos se conviertan en herramientas ideológicas que instrumentalicen a las personas más vulnerables.

\section{BIBLIOGRAFÍA}

- ADELL, N.; BENDIX, R. F.; BORTOLOTTO, C. ; TAUSCHEK, M. (2015) Between Imagined Communities and Communities of Practice: Participation, Territory and the Making of Heritage. En BENDIX, R. Göetingen Studies in Cultural Property. Göetingen: Universitätsverlag Göttingen, 2015, vol. 8

- ALONSO GONZÁLEZ, P. (2013) The Heritage Machine: A Heritage Ethnography in Maragatería (Spain). Tesis doctoral inédita, Universidad de León, 2013

- BENDIX, R.; EGGERT, A.; PESELMANN, A. (ed.) (2012) Heritage Regimes and the State. Göttingen: Universitätsverlag Göttingen, 2012

- BENHABIB, S. (2006) Las reivindicaciones de la cultura: igualdad y diversidad en la era global. Buenos Aires: Katz, 2006

- CoCA, A. (2008) El Plan de Desarrollo Sostenible del Parque Natural de los Alcornocales. En Los Camperos. Territorios, usos sociales y percepciones en un espacio natural andaluz. Sevilla: Fundación Blas Infante, 2008, pp. 557-575

- COOKE, B.; KOTHARI, U. (2001) Participation: The New Tyranny? New York: Zed Books, 2001

- CORNWALL, A. (2008) Unpacking 'Participation': models, meanings and practices. Community Development Journal, vol. 43, n. ${ }^{\circ} 3,2008$, pp. 269-283

- CORTÉS VAZQUEZ, J. A.; JIMÉNEZ-ESQUINAS, G.; SÁNCHEZ-CARRETERO, C. (en prensa). Heritage and Social Participation: critical approaches and new comparative strategies. Anthropology Today, en prensa

- CRIADO-BOADO, F. (1996) Hacia un modelo integrado de investigación y gestión del Patrimonio Histórico: la cadena interpretativa como propuesta. PH Boletín, vol. 16, 1996, pp. 73-78

- CRIADO-BOADO, F.; BARREIRO, D. (2013) El patrimonio era otra cosa. Estudios atacameños, vol. 45, 2013, pp. 5-18

- DAVALLON, J. (2010) The Game of Heritagization. En J. FRIGOLÉ; X. ROIGÉ (ed.) Constructing Cultural and Natural Heritage: Parks, Museums and Rural Heritage. Girona: ICRPC, 2010, pp. 39-62

- DELEUZE, G. (1994) Spinoza y las tres "Éticas". Nombres: Revista de Filosofía, vol. 4, n. ${ }^{\circ} 4$, 1994, pp. 95-108 
- DURÁN SALADO, I. (2007) Participación y percepción social en la gestión de conjuntos patrimoniales. Sevilla: IAPH. Laboratorio del Paisaje Cultural, 2007 <http://repositorio.iaph. es/bitstream/11532/163090/1/PERCEPCION SOCIAL EN CONJUNTO_PATRIMONIALES.pdf> [Consulta: 08/09/2016]

- FRASER, N. (1997) Iustitia Interrupta: Reflexiones críticas desde la posición 'postsocialista'. Santafé de Bogotá: Siglo del Hombre Editores, Universidad de los Andes. Facultad de Derecho, 1997

- HAFSTEIN, V. (2014) Cultural Heritage. En BENDIX, R.; HASAN-ROKEM, G. (ed.) A Companion to Folklore. Oxford: Wiley Blackwell, 2014

- HERTZ, E. (2015) Bottoms, Genuine and Spurious. En ADELL, N.; BENDIX, R. F.; BORTOLOTTO, C.; TAUSCHEK, M. (ed.) Between Imagined Communities and Communities of Practice: Participation, Territory and the Making of Heritage. Göttingen: Universitätsverlag Göttingen, 2015, vol. 8, pp. 2557

- JIMÉNEZ-ESQUINAS, G. (2014) Crafting and Affective Landscapes: heritagization processes of textile crafts and landscapes in Costa da Morte (Galicia, Spain). Tesis doctoral inscrita. Institute of Heritage Sciences (Incipit-CSIC) and University of the Basque Country (EHU)

- JIMÉNEZ-ESQUINAS, G.; SÁNCHEZ-CARRETERO, C. (2015) Mediaciones patrimoniales para relaciones incendiarias: El caso del Santuario da Virxe da Barca de Muxía. revista $P H$, vol. 88,2015 , pp. 2-8

- JULLIEN, F. (2010) Las transformaciones silenciosas. Barcelona: Bellaterra, 2010

- JEUDY, H. (2001) La machinerie patrimoniale. Paris: Sens et Tonka, 2001

- LOGAN, W. S.; REEVES, K. J. (ed) (2009) Places of Pain and Shame. Dealing with Difficult Heritage. Abingdon: Routledge, 2009

- LOWENTHAL, D. (2000) Stewarding the Past in a Perplexing Present. En Values and Heritage Conservation. Los Angeles: Getty Conservation Institute, 2000, pp. 18-25

- MACMILLAN, C. M. (2010) Auditing citizen engagement in heritage planning: The views of citizens. Canadian Public Administration, vol. 53, n. ${ }^{\circ}$ 1, 2010, pp. 87-106

- MESKELL, L. (2005) Negative Heritage and Past Mastering in Archaeology. Anthropological Quarterly, vol. 75, n. ${ }^{\circ} 3,2002$, pp. 557-574

- MUÑOZ, J. E. (2006) Feeling Brown, Feeling Down: Latina Affect, the Performativity of Race, and the Depressive Position. Signs, vol. 31, n. ${ }^{\circ} 3,2006$, pp. 675-688

- PRATS, L. (2005) Concepto y gestión del patrimonio local. Cuadernos de Antropología Social, vol. 21, 2005, pp. 17-35
- QUINTERO MORÓN, V. (2011) "El patrimonio pertenece a todos". De la universalidad a la identidad, ¿cuál es el lugar de la participación social? En ARRIETA URTIZBEREA, I. (ed.) Legitimaciones sociales de las políticas patrimoniales $y$ museísticas. Abadiño: Universidad del País Vasco, 2011, pp. 45-78

- SÁNCHEZ-CARRETERO, C. (2012) Hacia una Antropología del conflicto aplicada al patrimonio. En SANTAMARINA CAMPOS, B. C. (ed.) Geopolíticas patrimoniales. De culturas, naturalezas e inmaterialidades. Una mirada etnográfica. Valencia: Germania, 2012, pp. 195-210

- SMITH, L. (2006) Uses of heritage. London; New York: Routledge, Taylor \& Francis Group, 2006

- STRATHERN, M. (2000) The Tyranny of Transparency. British Educational Research Journal, vol. 26, n. ${ }^{\circ}$ 3, 2000, pp. 309-321

- STRATHERN, M. (2003) Audit Cultures : Anthropological Studies in Accountability, Ethics and the Academy. Hoboken: Routledge, 2003

- TUNBRIDGE, J.; ASHWORTH, G. (1996) Dissonant Heritage: The Management of the Past as a Resource in Conflict. New York: John Wiley \& Sons, 1996

- URBINATI, S. (2011) Communities, Groups and Individuals: the Bearers of the Intangible Cultural Heritage. What is the Role Attributed to These Subjects in the Convention for the Safeguarding of the Intangible Cultural Heritage? En CICCHETTI, A.; FROSINI, J. O.; GOLA, M. (ed.) Public Law and Regulation. Rimini: Università di Bologna \& Maggioli Edittore, 2011, vol. 6, pp. 163-189

- URBINATI, S. (2012) The Role for Communities, Groups and Individuals under the Covention for the Safeguarding of the Intangible Cultural Heritage. En BORELLI, S.; LENZERINI , F. (ed.) Cultural Heritage, Cultural Rights, Cultural Diversity: New Development in International Law. Leiden: Martinus Nijhoff, 2012, p. 41

- ŽıžEK, S. (2008) Violence. New York: Picador, 2008

\section{NOTA}

1. El trabajo que presentamos en este debate se enmarca dentro del proyecto $\mathrm{NEARCH}$ financiado por la programa CULTURA de la Comisión Europea y el proyecto de I+D del Plan Nacional, "ParticiPat, Patrimonio y participación social: propuesta metodológica y revisión crítica" (HAR2014-54869-R). 